\title{
Korrekturosteotomie fehlverheilter körperferner Speichenbrüche
}

\section{Karl-Josef Prommersberger, Marion Mühldorfer-Fodor, Thomas Pillukat}

\section{Zusammenfassung}

Die Ausheilung in Fehlstellung ist trotz aller Fortschritte in der Behandlung der distalen Radiusfraktur weiterhin eine häufige Komplikation, wobei zunehmend Fehlstellungen trotz operativer Frakturversorgung gesehen werden. Wenn auch eine Fehlstellung nicht zwangsläufig zu Beschwerden führt, so klagen doch viele Patienten mit einer fehlverheilten distalen Radiusfraktur über Bewegungseinschränkungen, Kraftverlust und Schmerzen. Durch eine wohlgeplante Korrekturosteotomie des Radius kommt es zu einer statistisch signifikanten Verbesserung der Funktion und zur Reduktion der Schmerzen. Das klinische Ergebnis ist umso besser, je anatomischer der Radius hergestellt werden kann.

\section{Einleitung}

2003 erschien bereits einmal eine Arbeit zum Thema „Korrekturosteotomien am distalen Radius“ von uns im OP-Journal [9]. Auch wenn sich naturgemäß weder an den Auswirkungen der Fehlstellung des distalen Radius noch an den Prinzipien der Radiuskorrekturosteotomie seither etwas geändert hat, so sehen wir uns doch heute zunehmend mit anderen Problemen konfrontiert als 2003.

Während damals fast ausnahmslos der Ausheilung in Fehlstellung eine konservative Behandlung mit sekundärem Korrekturverlust bei primär gutem Repositionsergebnis voranging, werden heute viele Korrekturoperationen nach primär operativ in unzureichender Stellung fi-

OP-JOURNAL 2011; 27: 228-233

(c) Georg Thieme Verlag KG Stuttgart · New York DOI http://dx.doi.org/10.1055/s-0031-1280468

\section{Corrective Osteotomy for Malunited Fractures of the Distal Radius}

In spite of all advances in the treatment of distal radius fractures, malunion of the distal radius is still a common complication. More and more we are faced with malunion following operative treatment of distal radius fractures. It may be true that not all non-anatomically aligned fractures of the distal radius lead to a poor clinical outcome. Nevertheless, many patients with a radial malunion complain about loss of function, reduced grip strength, and pain. Corrective radial osteotomy improves wrist and forearm function and reduces pain to a statistically significant degree. Patients with the least residual deformity after corrective osteotomy have significantly better outcomes than those with a gross residual deformity.
Bei extraartikulärer Fehlstellung findet sich meist eine Kombination aus einer Abflachung der Ulnarinklination des Radius und einer Verkippung der Radiuskonsole nach dorsal oder palmar als auch einer Verkürzung des Radius im Verhält-

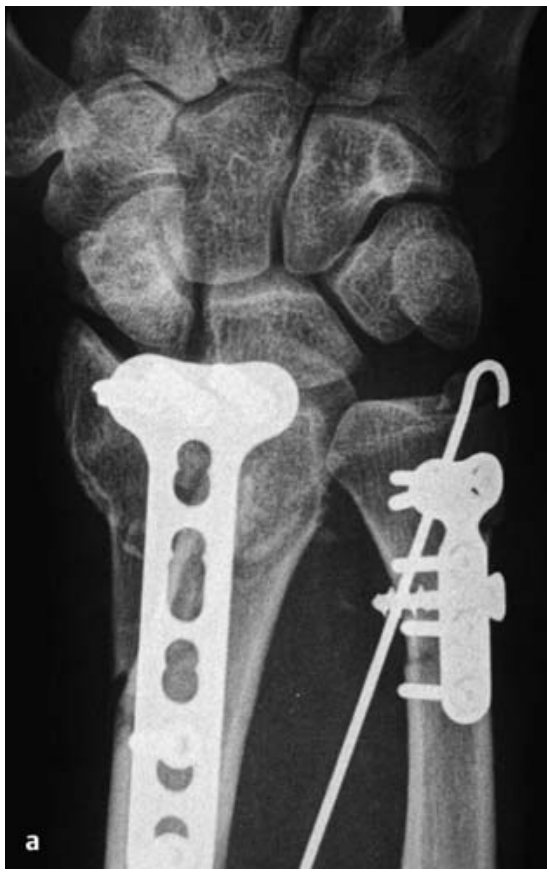

Entsprechend ist die Ausheilung in Fehlle Plattenosteosynthese erzielten Fortschritte weiterhin eine häufige, vielleicht sogar die häufigste Komplikation der distalen Radiusfraktur.

\section{Art der Fehlstellung}

Am unverletzten, physiologisch konfigurierten Radius ist die distale Gelenkfläche $22^{\circ}$ nach ellenseits und $10^{\circ}$ nach beugeseits geneigt, wobei bei $90 \%$ der Bevölkerung Radius und Ulna gleich lang sind, also eine Ulna-null- oder Ulna-neutral-Varianz vorliegt.

Heilt der distale Radius nach einer Fraktur in Fehlstellung aus, gilt es zwischen extraartikulären und intraartikulären sowie kombiniert extra-/intraartikulären Fehlstellungen zu unterscheiden. 


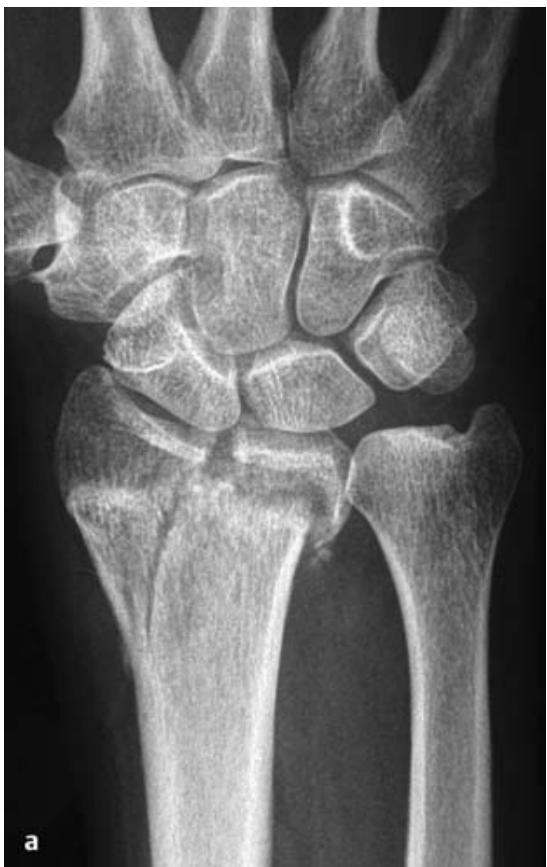

Abb. 2a Mit Spalt- und zugleich Stufenbildung fehlverheilte intraartikuläre Radiusfraktur eines 57-jährigen Mannes.

nis zur Ulna. In $50 \%$ der Fälle besteht zusätzlich ein Drehfehler des distalen Radius gegenüber dem Radiusschaft [7]. Des Weiteren findet sich gelegentlich ein Versatz der Radiuskonsole nach dorsal oder palmar und/oder radial.

Intraartikuläre Fehlstellungen beobachtet man in Form von Gelenkstufen oder Spaltbildungen (Abb.2a-g). Diese können die radiokarpale Gelenkfläche oder die Incisura ulnaris des Radius betreffen. Unter biomechanischen und indikatorischen Gesichtspunkten ist es wichtig, dass man sich bewusst macht, dass neben der direkten Inkongruenz des körperfernen Drehgelenks zwischen Elle und Speiche (= distales Radioulnargelenk) aufgrund einer Stufen- oder Spaltbildung in der Incisura ulnaris radii auch jede extraartikuläre Fehlstellung des distalen Radius die Geometrie des distalen Radioulnargelenks beeinträchtigt und somit Auswirkungen auf die Unterarmdrehung hat.

\section{Symptomatik der fehlverheilten distalen Radiusfraktur}

Nicht jede fehlverheilte Fraktur des distalen Radius führt zu einer Funktionsbeeinträchtigung. In zahlreichen experimentellen Untersuchungen konnte jedoch gezeigt werden, dass eine strenge Korrelation zwischen der Form des dis-

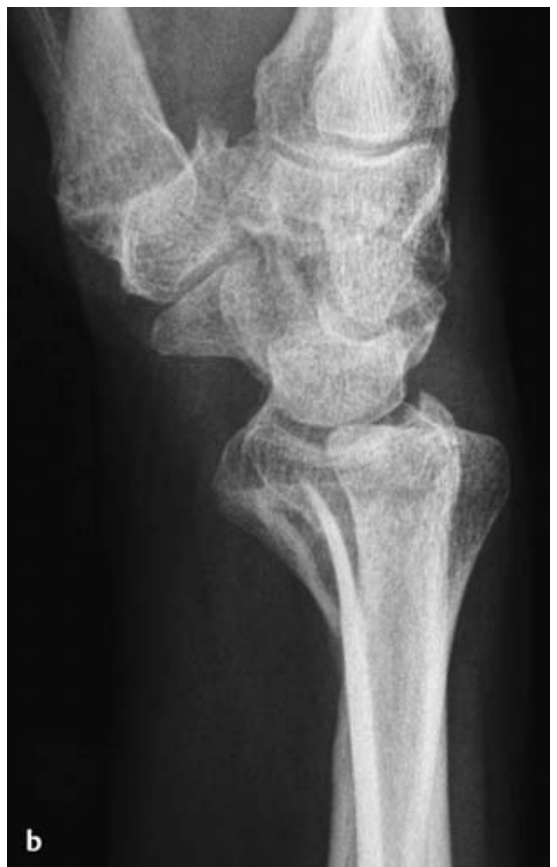

Abb. 2b Die Seitaufnahme zeigt einen zusätzlichen Versatz des distalen Fragments nach palmar.

talen Radius und der Funktion des Handgelenks besteht.

Viele, wenn auch nicht alle Patienten klagen entsprechend über eine Einschränkung der Handgelenksextension/-flexion und der Unterarmdrehung sowie einen Kraftverlust und über Schmerzen.

Die Schmerzen sind hauptsächlich am Ellenkopf lokalisiert. Dies ist für die Patienten oft schwer nachzuvollziehen, war doch die Speiche und nicht die Elle gebrochen, biomechanisch jedoch aufgrund des Ulna-Impaktionssyndroms bei posttraumatischer Ellen-plus-Variante leicht zu erklären. Geklagt wird auch, und dies nicht nur von Frauen, sondern auch von Männern, über das gestörte Erscheinungsbild des Handgelenks mit einem prominenten Ellenkopf, typisch für eine in Extensionsstellung fehlverheilte Radiusfraktur, und einer Bajonettdeformität, typisch für eine mit vermehrter Flexionsstellung der Radiusgelenkfläche verheilten Fraktur. Auch wenn Patienten mit einer fehlverheilten distalen Radiusfraktur selten über Beschwerden seitens eines Karpaltunnelsyndroms berichten, konnten wir doch mittels subtiler neurophysiologischer Untersuchungen in einem hohen Maße Irritationen des $\mathrm{N}$. medianus nachweisen.

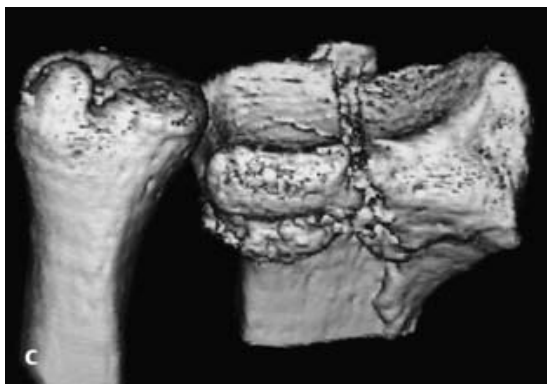

Abb. 2c In der 3-D-CT-Rekonstruktion kommen Spalt- und Stufenbildung als auch der palmare Versatz und die Verkürzung des Radius im Verhältnis zur Ulna zur Darstellung.

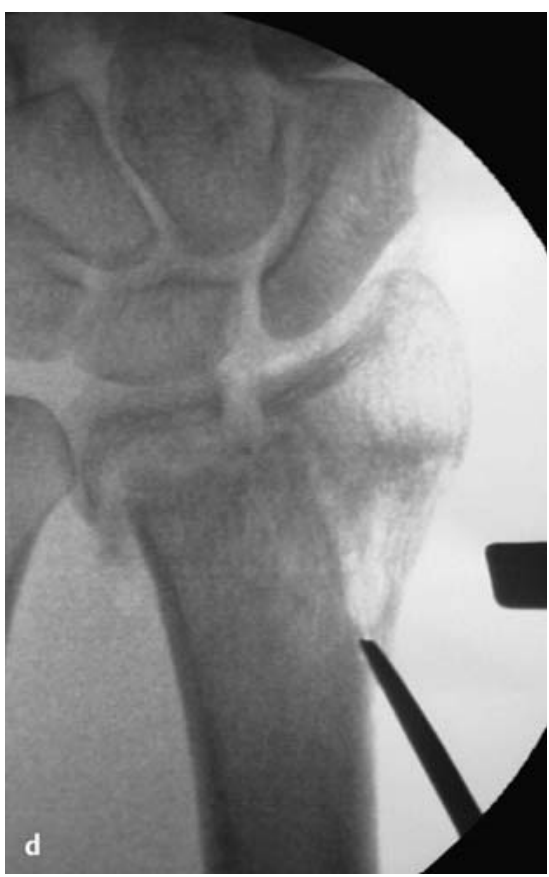

Abb.2d Intraoperative Durchleuchtung: Aufsuchen des ehemaligen Frakturspalts unter Bildwandlerkontrolle mit dem Meißel.

\section{Einfluss der Fehlstellung des distalen Radius auf die Biomechanik des Handgelenks}

Die vorgenannten Beschwerden der Patienten lassen sich leicht mittels der durch die Fehlstellung verursachten Störungen der Biomechanik des Handgelenks erklären [8].

Da der distale Radius sowohl an der Bildung des proximalen Handgelenks als auch des distalen Radioulnargelenks beteiligt ist, wirkt sich die posttraumatische Fehlstellung direkt auf diese beiden Gelenke aus. Das Gelenk zwischen der körpernahen und körperfernen Handwurzelreihe (= Mediokarpalgelenk) ist hingegen nur indirekt von der Fehlstellung des distalen Radius betroffen. Darü- 


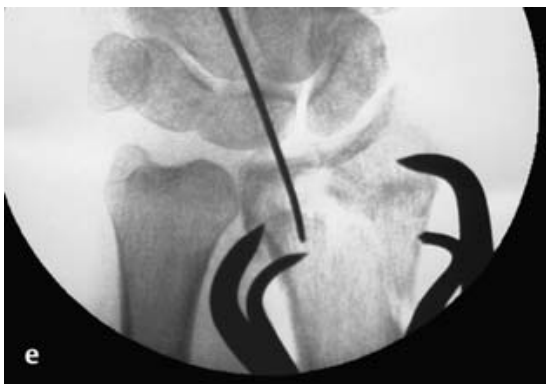

Abb.2e Intraoperative Durchleuchtung: Spalt- und Stufenbildung wurden ebenso wie das Längenverhältnis ausgeglichen. Provisorische Fixation mit Repositionszangen.

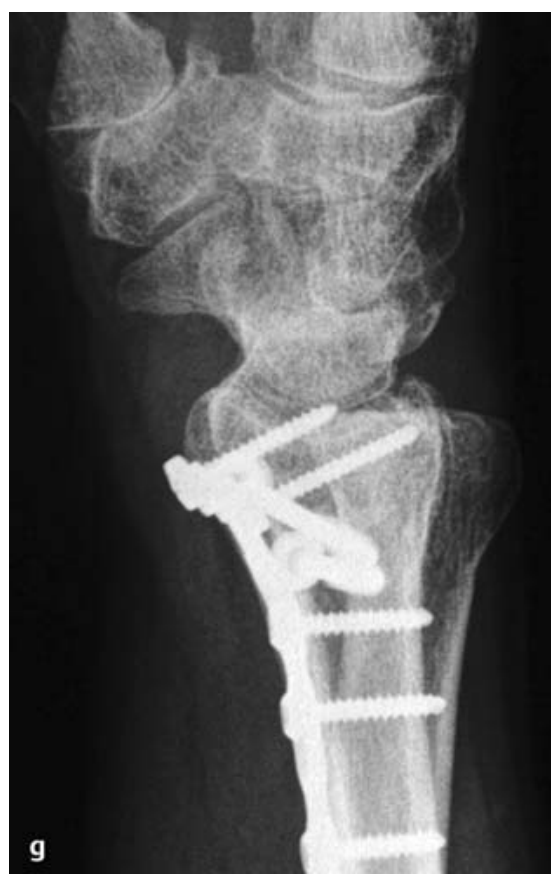

Abb.2g Geringfügige Kippung der Radiusgelenkfläche frühpostoperativ in der Seitaufnahme nach dorsal.

ber hinaus beeinträchtigt der deform verheilte Radius die Weichteile, insbesondere den $\mathrm{N}$. medianus und die über ihn hinwegziehenden Sehnen-MuskelEinheiten.

Die Kraftübertragung erfolgt am unverletzten, gesunden Handgelenk zu 15$30 \%$ über die Ulna und zu 70-85\% über den Radius. Am Radius verteilt sich die Kraftübertragung im Verhältnis 60:40 auf die Fossa scaphoidea und die Fossa lunata. Die Fehlstellung des distalen Radiusfragments beeinflusst sowohl die Kraftübertragung am gesamten Handgelenk als auch die Druckverteilung am Radius. Bei Dorsalkippung des distalen Radiusfragments kommt es unter Verschmälerung der Belastungszone zu einer Verlagerung der Belastungszone

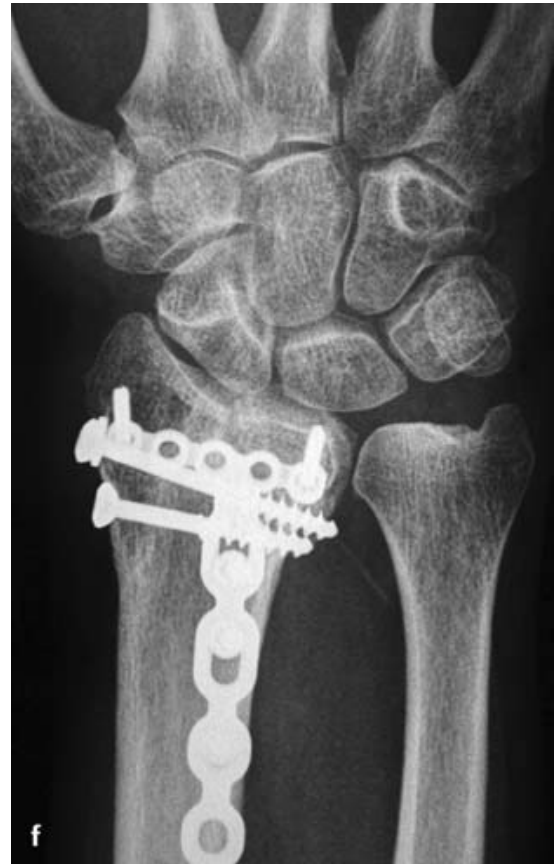

Abb. $2 f$ Das frühpostoperative Röntgenbild in Draufsicht zeigt noch eine verbliebene geringe Stufe der Radiusgelenkfläche. Der Radius ist ansonsten in Form und Länge gut wiederhergestellt.

am Radius nach dorsal. Sowohl die Dorsalkippung der Radiusgelenkfläche, mehr jedoch noch die Verkürzung des Radius bewirken darüber hinaus einen dramatischen Druckanstieg ulnokarpal.

Die Verkippung des distalen Radius nach dorsal oder palmar und nach radial führt sowohl zu Veränderungen der physiologischen Stellung der radiokarpalen Gelenkfläche als auch zur gleichzeitigen Verkippung der Incisura ulnaris gegenüber dem unveränderten Ulnakopf als Gelenkpartner und somit zu einer Inkongruenz im distalen Radioulnargelenk. So vermindert sich die Kontaktfläche im distalen Radioulnargelenk in Neutralstellung des Unterarms bei einer Radiusverkürzung von $1 \mathrm{~mm}$ auf $77 \%$ des Normalwerts und bei $2 \mathrm{~mm}$ auf $58 \%$. Die Dorsalkippung des distalen Radiusfragments von $5^{\circ}$ verursacht eine Reduktion der Kontaktfläche im distalen Radioulnargelenk auf 55\%; eine vermehrte Palmarkippung von $5^{\circ}$ gar auf $29 \%$. Neben dieser Inkongruenz im distalen Radioulnargelenk kommt es durch die Verkippung des distalen Radiusfragments und mehr noch durch die Verkürzung des Radius gegenüber der Ulna zu einem Spannungsanstieg des dreieckförmigen Faserknorpels ellenseitig im Handgelenk $(=$ ulnokarpaler Komplex = TFCC $)$. Sowohl die Inkongruenz im distalen Radioulnargelenk als auch der Spannungs- anstieg des TFCC wirken sich negativ auf die Unterarmdrehung aus.

Die Fehlstellung der Radiusgelenkfläche wirkt sich sowohl auf die proximale als auch indirekt auf die distale Handwurzelreihe aus. Bei den meisten mit einer Dorsalkippung fehlgeheilten Frakturen folgt das Lunatum dem Radius in die Fehlstellung und verkippt nach dorsal. Um eine Neutralstellung der Hand trotz Verkippung von Radius und Lunatum zu ermöglichen, wird das Kapitatum kompensatorisch flektiert, sodass im seitlichen Röntgenbild eine Zickzack-Deformität der Handwurzel resultiert. Deutlich seltener beobachtet man eine Parallelverschiebung der gesamten Handwurzel ohne Verkippung in sich nach dorsal im Verhältnis zur Radiuslängsachse.

\section{Behandlungsoptionen}

Bei der Entscheidung, ob eine Behandlung notwendig ist oder nicht, sind vorrangig der Patient und seine Bedürfnisse zu berücksichtigen. Welche Behandlungsmaßnahme man ergreift, hängt hingegen ab von der Art und dem Ausmaß der Fehlstellung, dem Umfang der Funktionseinschränkung und davon, ob bereits arthrotische Veränderungen vorhanden sind.

Neben Eingriffen zur Wiederherstellung der „korrekten“ Position der Radiusgelenkfläche im Verhältnis zur Handwurzel und dem Ellenkopf gibt es Eingriffe, die der Funktionsverbesserung, insbesondere der Verbesserung der Unterarmdrehung, dienen ohne Wiederherstellung der „normalen“ Anatomie und solche, deren ausschließliches Ziel die Schmerzreduktion ist. Bei fehlverheilten distalen Radiusfrakturen zeigt sich nach unserer Erfahrung die Handgelenksdenervation sehr effektiv, ohne dass es zu einem Verlust der Beweglichkeit kommt. Bei stärkeren Schmerzen und einer Radiokarpalarthrose sind die radio-skapho-lunäre Fusion oder gar die vollständige Handgelenksversteifung zu diskutieren, wobei die Schmerzreduktion mit einer Bewegungseinschränkung erkauft wird [2]. Eingriffe mit dem Ziel der Funktionsverbesserung ohne Wiederherstellung der „normalen“ Anatomie betreffen im Wesentlichen die Ellenseite des Handgelenks und dienen in erster Linie der Verbesserung der Unterarmrotation. Gute Erfahrung haben wir hierbei mit Hemiresektionsarthroplastik nach Bowers gemacht [13]. Wenn irgend möglich, bevorzugen wir heute den Ersatz des 


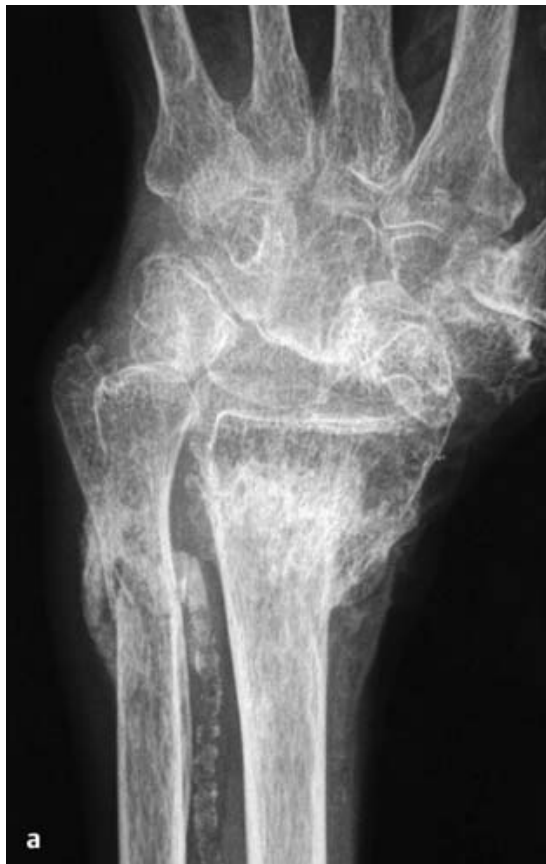

Abb. 3a Kontraindikation gegen eine Radiuskorrekturosteotomie trotz erheblicher Verkürzung und Aufhebung der Ulnarinklination des distalen Radius bei massiver Osteoporose, erheblichen arteriosklerotischen Verkalkungen und Derangierung des Karpus.

Ulnakopfs durch eine Prothese [14]. Als Eingriffe zur Wiederherstellung „normaler" anatomischer Verhältnisse sind Ulnaverkürzungsosteotomie und Radiuskorrekturosteotomie zu nennen.

\section{Radiuskorrekturosteotomie}

Bei der Indikationsstellung zur Radiuskorrekturosteotomie sind primär die vorhandenen Beschwerden ausschlaggebend. Die Fehlstellung im Röntgenbild ist von sekundärer Bedeutung.

Zu berücksichtigen sind: die Funktionseinschränkung, das Ausmaß der Schmerzen, ob eine mediokarpale Instabilität vorliegt, ob Probleme seitens des DRUGes bestehen und auch das gestörte ästhetische Erscheinungsbild des Handgelenks. Radiuskorrekturosteotomie und Ulnaverkürzung sind dabei nicht als Alternativen $\mathrm{zu}$ verstehen, denn jede Verkippung des distalen Radius führt zu einer Inkongruenz des distalen Radioulnargelenks.

Entsprechend ist nur bei Kippungen des Radiusgelenkblocks von weniger als $10^{\circ}$ nach dorsal und weniger als $20^{\circ}$ nach palmar eine Ulnaverkürzung indiziert [10].

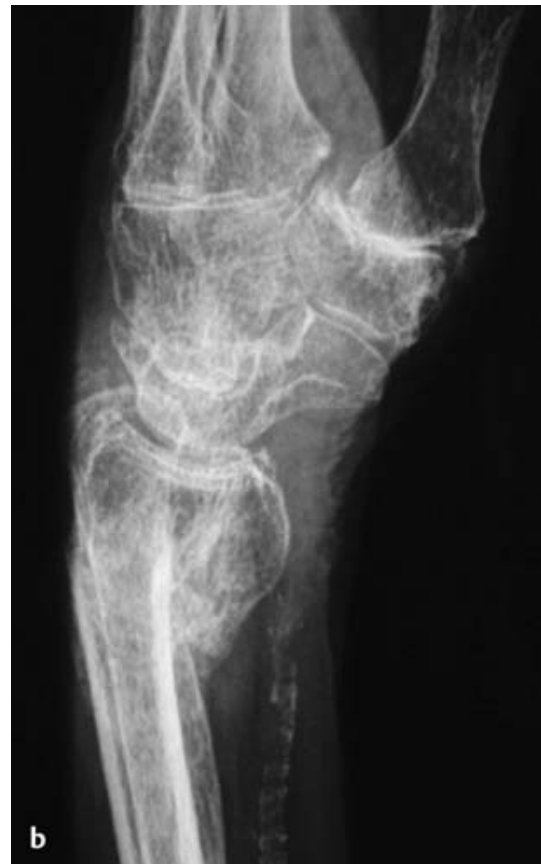

Abb. 3b Auch im seitlichen Röntgenbild zeigt sich eine Fehlstellung des distalen Radius mit vermehrter Palmarkippung der Radiusgelenkfläche. Wiederum ist die erhebliche Arteriosklerose sowie eine Arthrose des Daumensattelgelenks zu erkennen.

Verkürzungen des Radius um mehr als $12 \mathrm{~mm}$ erfordern meist eine zuklappende Radiuskorrekturosteotomie in Kombination mit einer Ulnaverkürzung.

Absolute Kontraindikationen gegen eine Radiuskorrekturosteotomie sind: ein schlechter Allgemeinzustand, degenerative Veränderungen des Radiokarpalgelenks (Abb. $\mathbf{3} \mathbf{a}$ und $\mathbf{b}$ ) und eine fixierte Fehlstellung der Handwurzel, korrigiert diese doch nicht mit dem Radius und entsprechend persistieren trotz der Korrekturosteotomie die Beschwerden.

Finden sich Zeichen eines akuten komplexen regionalen Schmerzsyndroms, ist der Eingriff trotzdem indiziert, sofern die Fehlstellung und z.B. die hierdurch bedingte Irritation des $\mathrm{N}$. medianus als auslösendes Moment gesehen werden. Eine abgelaufene Algodystrophie ist nicht als Kontraindikation zu werten, allerdings sollte die Fingerbeweglichkeit möglichst nicht mehr eingeschränkt sein. Aufgrund der winkelstabilen Implantate spielt heute die Knochenqualität eine untergeordnete Rolle, sodass auch eine Osteoporose keine absolute Kontraindikation mehr darstellt.

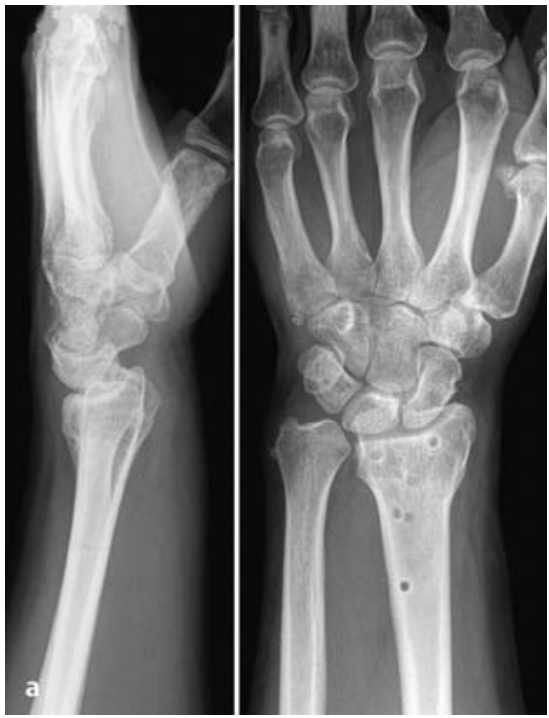

Abb.4a Trotz Plattenosteosynthese in erheblicher Fehlstellung mit Dorsalkippung der Radiusgelenkfläche von $40^{\circ}$ und mit Verkürzung von mehreren Millimetern ausgeheilte Radiusfraktur eines 44-jährigen Mannes. Die Entfernung des Osteosynthesematerials ist bereits erfolgt.

Weder eine Instabilität noch eine Arthrose des distalen Radioulnargelenks sind als Kontraindikation gegen eine Radiuskorrekturosteotomie zu betrachten, erfordern jedoch gegebenenfalls gleichzeitig oder sekundär einen Eingriff auf der Ellenseite des Handgelenks.

Bei einer Pseudarthrose des Ellengriffelfortsatzes konnten wir mehrfach nach einer Radiuskorrektur eine Ausheilung beobachten, ohne dass ein Eingriff auf der Ellenseite erfolgte.

Auch ein fortgeschrittenes Alter gilt nicht länger als Kontraindikation.

Wir konnten zeigen, dass auch ältere Patienten statistisch signifikant von einer Radiuskorrektur profitieren, wenn auch nicht im gleichen Umfang wie jüngere Patienten [5]. Aufgrund der guten spontanen Korrekturpotenz des distalen Radius ist im Kindesalter die Indikation zu einer Radiuskorrekturosteotomie nur gegeben bei partiellem oder vollständigem Wachstumsstopp oder falls die verbleibende Zeit bis zum Wachstumsende keine ausreichende Korrektur erwarten lässt.

Wie die Ergebnisse eines Vergleichs frühzeitig versus später (>10 Monate nach dem Unfall) durchgeführter Korrekturosteotomien zeigen, spricht nichts dagegen, die Radiuskorrekturosteotomie vorzunehmen, sobald klar wird, dass die 


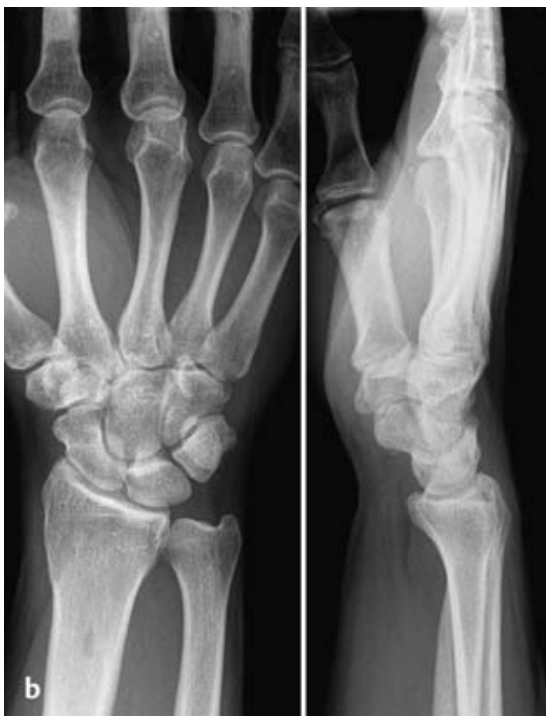

Abb.4b Röntgenaufnahme der unverletzten Gegenseite.

Indikation gegeben ist, die Schwellung rückläufig und die Fingerbeweglichkeit ordentlich ist [3].

Zur Planung des Eingriffs ist es erforderlich, Röntgenaufnahmen beider Handgelenke in 2 Ebenen zu fertigen (Abb. $\mathbf{4 a}$ und $\mathbf{b}$ ).

Eine Computertomografie ist indiziert bei Verdacht auf einen Rotationsfehler des distalen Fragments und auf Arthrose des distalen Radioulnargelenks [7]. Übersteigt die Kippung des Mondbeins nach dorsal die Dorsalkippung der Radiusgelenkfläche, muss man den Verdacht auf eine begleitende skapholunäre Dissoziation hegen. Eine Handgelenksspiegelung oder Kernspinuntersuchung mit Kontrastmittel ist dann ebenso wie zur Abklärung der Knorpelsituation bei geplanter intraartikulärer Korrekturosteotomie sinnvoll.

Auf der Basis des Vergleichs der radiologischen Messdaten (Neigung der Radiusgelenkfläche in der Seitaufnahme, Neigung der Radiusgelenkfläche in der Draufsicht, Verkürzung des Radius im Verhältnis zur Elle) der gesunden, unverletzten Seite und der verletzten Seite ist der Korrektureingriff zeichnerisch zu planen, wobei heute zunehmend die Planung mithilfe des Computers erfolgt [1].

Bei der Wahl des Zugangs für die Radiuskorrekturosteotomie gilt es zu berücksichtigen, ob gleichzeitig neben der Fehlstellung des distalen Radius eine korrekturbedürftige karpale Instabilität vorliegt und synchron ein Eingriff am dis-

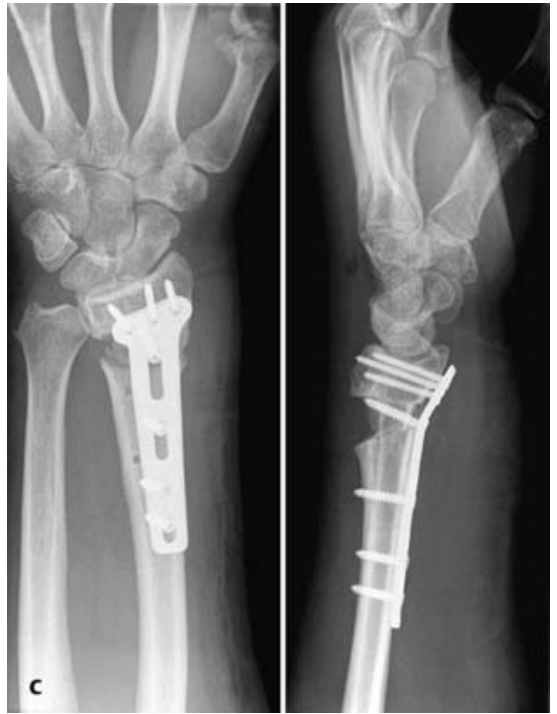

Abb.4c Frühpostoperative Situation: Der Radius ist in Form und Länge wieder hergestellt. Der eingebrachte Beckenkammblock wurde mittels einer Hohlfräse mit einem Durchmesser von $2 \mathrm{~cm}$ entnommen. Er füllt den Osteotomiespalt nicht vollständig ist.

talen Radioulnargelenk oder eine Entfernung noch einliegenden Osteosynthesematerials erforderlich ist. Rotationsfehler lassen sich erfahrungsgemäß besser von der Beugeseite erkennen und korrigieren. Ansonsten gilt, dass in vermehrter Flexionsstellung fehlverheilte Speichenbrüche der Korrektur von der Beugeseite bedürfen, wohingegen mit Dorsalkippung fehlgeheilte Radiusfrakturen von dorsal, radial und palmar korrigiert werden.

Heute, da frische distale Radiusfrakturen mit Dorsalkippung der Radiusgelenkfläche überwiegend mittels winkelstabiler Plattenosteosynthese von palmar versorgt werden, gewinnt die Korrekturosteotomie fehlverheilter Extensionsfrakturen von palmar zunehmend an Popularität [11].

Es ist zwar technisch einfacher, die Osteotomie proximal der ursprünglichen Fraktur vorzunehmen, dies kann jedoch zu erheblichen Problemen führen. So kann es zu Subluxationen im distalen Radioulnargelenk und einer Knickbildung des Radius am Übergang der Metazur Diaphyse (Humpback-Deformität) kommen. Letztere führt zu einer gestörten Druck- und Kraftübertragung von der Hand auf den Unterarm mit der Gefahr der Refraktur bei Entfernung des Osteosynthesematerials. Von daher ist es ratsam, die Osteotomie weit distal im Bereich der ursprünglichen Fraktur anzulegen.

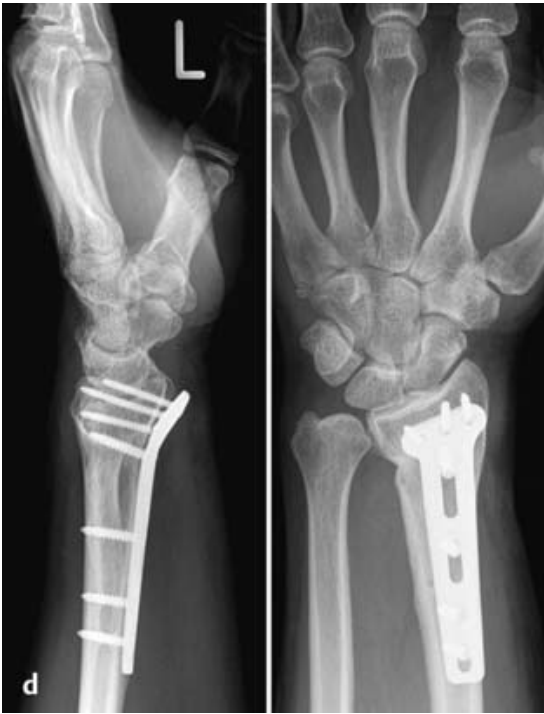

Abb. 4d Nach wenigen Wochen ist der Knochenblock vollständig integriert und der Osteotomiespalt bereits fast komplett mit Knochen aufgefüllt. Bei dem sehr kräftigen Patienten ist es trotz Verwendung eines sehr stabilen, winkelstabilen, speziell für die Radiuskorrekturosteotomie von uns entwickelten Implantats und trotz Interposition eines bikortikalen Knochenblocks in den Osteotomiespalt zu einem leichten Korrekturverlust gekommen. Ausdruck dafür ist die Prominenz der Platte distal-palmar und der Umstand, dass eine Schraube gebrochen ist. Dies unterstreicht die Bedeutung der Wahl des Osteosynthesematerials und des Umgangs mit dem Osteotomiespalt insbesondere bei länger bestehenden Fehlstellungen und sehr kräftigen Männern.

Bedarf es keiner Verlängerung des Radius, was nach unserer Erfahrung oft bei fehlverheilten Flexionsfrakturen der Fall ist, muss man den Radius nicht vollständig durchtrennen, sondern kann die Gegenkortikalis und/oder das Periost der Gegenseite als eine Art Scharnier belassen. In diesen Fällen resultiert ein dreieckförmiger Osteotomiespalt. Bedarf es aber der Verlängerung des Radius, ist eine komplette Durchtrennung erforderlich. Dann resultiert ein trapezförmiger, bzw. sofern es auch die Korrektur der Ulnarinklination erfordert, ein doppelttrapezförmiger Osteotomiespalt.

Was nun tun mit dem Osteotomiespalt? In der überwiegenden Mehrzahl der Fälle wird er immer noch aufgefüllt; eher selten mit Knochenersatzmaterial, bei Verwendung einer winkelstabilen Platte zur Fixation zunehmend häufiger nur mit Spongiosa, am häufigsten jedoch immer noch mit einem bi- oder trikortikalen Knochenblock, in der Regel vom Beckenkamm entnommen (Abb. 4c und d). 
Im Grunde können zur Fixation des Radius nach einer Korrekturosteotomie alle Osteosyntheseverfahren verwandt werden, wie sie auch zur Versorgung frischer Radiusfrakturen zur Anwendung kommen: die Kirschner-Draht-Spickung, die Plattenosteosynthese, der Fixateur externe. Bei lange bestehenden Fehlstellungen und bei kräftigen Patienten lastet ein enormer Druck auf der Osteosynthese, weshalb es ratsam ist, eine sehr stabile Platte $\mathrm{zu}$ verwenden, um ein Implantatversagen zu vermeiden $[4,6]$.

Zur Korrekturosteotomie fehlverheilter Radiusfrakturen gibt es mehr als 200 Veröffentlichungen.

Alle Veröffentlichungen, die sich nicht nur ausschließlich mit der Operationstechnik beschäftigen, belegen, dass es durch die Korrektur der Fehlstellung des distalen Radius zu einer Verbesserung der Handgelenksbeweglichkeit, der Unterarmdrehung und der groben Kraft und zugleich zu einer Verminderung der Schmerzen kommt.

Die Änderungen von prä- zu postoperativ sind dabei signifikant. Zugleich zeigt sich - wie auch bei frischen Radiusfrakturen - ein klarer Zusammenhang zwischen dem Ergebnis im Röntgenbild und dem klinischen Resultat. Je anatomischer der Radius rekonstruiert werden konnte, desto besser das klinische Ergebnis [12].
Die wohl gravierendste Komplikation nach einer Radiuskorrekturosteotomie ist die Entwicklung einer Pseudarthrose des Radius. Im eigenen Krankengut mussten wir sie vor Verwendung winkelstabiler Implantate in $1 \%$ der Fälle beobachten [6]. Die Rate von Radiuspseudarthrosen bei frischen Frakturen lag damals bei $0,1 \%$. Seit Einführung winkelstabiler Implantate spielen Pseudarthrosen sowohl bei frischen Frakturen als auch nach Korrekturosteotomien zahlenmäßig keine Rolle mehr.

\section{Literatur}

${ }^{1}$ Athwal GS, Ellis RE, Small CF et al. Computerassisted distal radius osteotomy. J Hand Surg [Am] 2003; 28: 951-958

2 Beyermann K, Prommersberger K-J, Krimmer $\mathrm{H}$ et al. Radio-scapho-lunate fusion as treatment of posttraumatic radiocarpal arthrosis. Eur J Trauma 2000; 26: 169-175

${ }^{3}$ Jupiter JB, Ring D. A comparison of early and late reconstruction of malunited fractures of the distal end of the radius. J Bone Joint Surg [Am] 1996; 78: 739-748

${ }_{4}$ Müller LP, Klitscher D, Rudig L et al. Locking plates for corrective osteotomy of malunited dorsally tilted distal radial fractures: a biomechanical study. J Hand Surg [Br] 2006; 31: 556-561

5 Pillukat T, van Schoonhoven J, Prommersberger $\mathrm{K}-\mathrm{J}$. Ist die Korrekturosteotomie der fehlverheilten distalen Radiusfraktur auch beim älteren Menschen indiziert? Handchir Mikrochir Plast Chir 2007; 39: 71-77

${ }^{6}$ Prommersberger K-J, Fernandez DL. Nonunion of distal radius fractures. Clin Orthop Relat Res 2004; 419: 51-56

7 Prommersberger K-J, Fröhner SC, Schmitt RR et al. Rotational deformity in malunited fractures of the distal radius. J Hand Surg [Am] 2004; 29: 110-115
8 Prommersberger $K-J$, Lanz $U$. Biomechanik der fehlverheilten distalen Radiusfraktur. Handchir Mikrochir Plast Chir 1999; 31: 221-226

9 Prommersberger K-J, Lanz U. Korrekturosteotomien. OP-Journal 2003; 19: 58-64

10 Prommersberger K-J, van Schoonhoven J. Störungen des distalen Radioulnargelenkes nach distaler Radiusfraktur. Unfallchirurg 2008; 111: 173-186

11 Prommersberger K-J, van Schoonhoven J. Korrekturosteotomie der fehlverheilten Extensionsfraktur des distalen Radius von palmar Unfallchirurg 2007; 110: 631-636

12 Prommersberger K-J, van Schoonhoven J, Lanz $U$. Outcome after corrective osteotomy for malunited fractures of the distal end of the radius. J Hand Surg [Br] 2002; 27: 55-60

${ }^{13}$ Schober F, van Schoonhoven J, Prommersberger $K$-J et al. Die Hemiresektions-Interpositionsarthroplastik nach Bowers zur Behandlung der posttraumatischen Arthrose des distalen Radioulnargelenks nach distaler Radiusfraktur. Handchir Mikrochir Plast Chir 1999; 31: 378-382

${ }^{14}$ van Schoonhoven J, Prommersberger K-J. Die Ulnakopfprothese. Unfallchirurg 2008; 111: 187-192

Prof. Dr. med.

Karl-Josef Prommersberger

Chefarzt

Dr. med. Marion Mühldorfer-Fodor

Oberärztin

Dr. med. Thomas Pillukat

Oberarzt

Klinik für Handchirurgie

Rhön-Klinikum AG

Salzburger Leite 1

97615 Bad Neustadt

hael@handchirurgie.de 